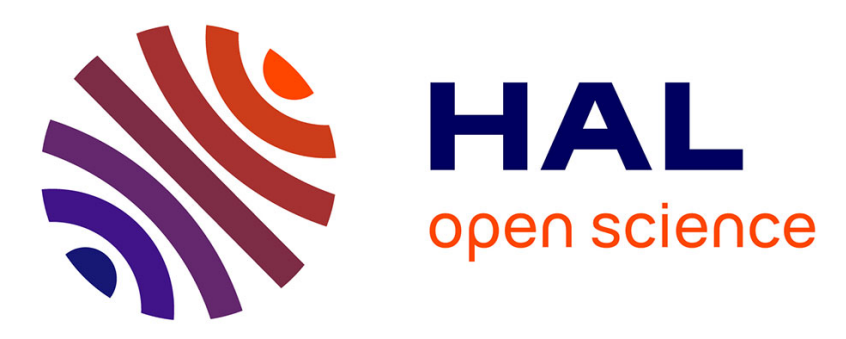

\title{
Efficient On-The-Fly Emptiness Check for Timed Büchi Automata
}

\author{
Frédéric Herbreteau, B. Srivathsan
}

\section{To cite this version:}

Frédéric Herbreteau, B. Srivathsan. Efficient On-The-Fly Emptiness Check for Timed Büchi Automata. ATVA - 8th International Symposium on Automated Technology for Verification and Analysis - 2010, Sep 2010, Singapore, Singapore. pp.218-232, 10.1007/978-3-642-15643-4_17 . inria-00496366

\section{HAL Id: inria-00496366 https://hal.inria.fr/inria-00496366}

Submitted on 30 Jun 2010

HAL is a multi-disciplinary open access archive for the deposit and dissemination of scientific research documents, whether they are published or not. The documents may come from teaching and research institutions in France or abroad, or from public or private research centers.
L'archive ouverte pluridisciplinaire HAL, est destinée au dépôt et à la diffusion de documents scientifiques de niveau recherche, publiés ou non, émanant des établissements d'enseignement et de recherche français ou étrangers, des laboratoires publics ou privés. 


\title{
Efficient On-The-Fly Emptiness Check for Timed Büchi Automata
}

\author{
F. Herbreteau and B. Srivathsan \\ LaBRI (Université de Bordeaux - CNRS)
}

\begin{abstract}
The Büchi non-emptiness problem for timed automata concerns deciding if a given automaton has an infinite non-Zeno run satisfying the Büchi accepting condition. The solution to this problem amounts to searching for a cycle in the so-called zone graph of the automaton. Since non-Zenoness cannot be verified directly from the zone graph, additional constructions are required. In this paper, it is shown that in many cases, non-Zenoness can be ascertained without extra constructions. An on-the-fly algorithm for the non-emptiness problem, using an efficient non-Zenoness construction only when required, is proposed. Experiments carried out with a prototype implementation of the algorithm are reported and the results are seen to be promising.
\end{abstract}

\section{Introduction}

Timed automata [1] are finite automata extended with clocks. The clock values can be compared with constants and reset to zero on the transitions, while they evolve continuously in the states. The emptiness problem for timed automata is decidable. Consequently, timed automata are used in tools like Uppaal [3] and Kronos [8] for model-checking reachability properties of real-time systems. They have also been successfully used for industrial case studies, e.g. [4].

For the verification of liveness properties, timed automata with Büchi conditions have been considered. The emptiness problem demands if there exists a non-Zeno run that visits an accepting state infinitely often. A run is non-Zeno if the total time elapsed during the run is unbounded. This further requirement makes the problem harder than classical Büchi emptiness.

The solution proposed in [1] relies on a symbolic semantics called the region graph. The emptiness problem for timed Büchi automata is reduced to classical Büchi emptiness on the region graph with an additional time progress requirement. However, the region graph construction is very inefficient for model checking real life systems. Zones are used instead of regions in the aforementioned tools. The zone graph is another symbolic semantics that is coarser than the region graph. Although it is precise enough to preserve reachability properties, it is too abstract to directly infer time progress and hence non-Zenoness.

Two approaches have been proposed in order to ascertain time progress. In [16], the automaton is transformed into a so-called strongly non-Zeno automaton, which has the property that all the runs visiting an accepting state 
infinitely often are non-Zeno. Hence, non-Zenoness is reduced to Büchi emptiness. However it has been proved in [12] that this construction sometimes results in an exponential blowup.

A careful look at the conditions for Zenoness yields another solution to verify time progress. Two situations can prevent time from diverging on a run. Either the value of a clock is bounded and not reset anymore, or some clock is reset and later checked to zero preventing time elapse in-between. In [12] a guessing zone graph has been introduced to identify clear nodes where time can elapse despite zero-checks. Hence timed Büchi automata emptiness reduces to Büchi emptiness on the guessing zone graph with two infinitary conditions namely clear nodes and accepting nodes. The guessing zone graph is only $|X|+1$ times bigger than the zone graph, where $|X|$ stands for the number of clocks. The straightforward algorithm handling both the above mentioned situations explores the guessing zone graph $\mathcal{O}(|X|)$ number of times.

On a parallel note, the emptiness problem for (untimed) Büchi automata has been extensively studied (see [13] for a survey). The best known algorithms in the case of multiple Büchi conditions are inspired by the Tarjan's SCC algorithm. An on-the-fly algorithm which computes the graph during the search, and terminates as soon as a satisfactory SCC is found, is crucial in the timed settings as the zone graph is often huge. It is known that the Couvreur's algorithm [9] outperforms all on-the-fly SCC-based algorithms.

In this paper, we present an on-the-fly algorithm that solves the emptiness problem for timed Büchi automata. We noticed from several experiments that Büchi emptiness can sometimes be decided directly from the zone graph, i.e. without using any extra construction. This is the way we take here. The Couvreur's algorithm is employed to search for an accepting SCC directly on the zone graph. Then, we face two challenges.

The first challenge is to detect if this SCC has blocking clocks, that is clocks that are bounded from above but are not reset in the transitions of the SCC. The SCCs with blocking clocks have to be re-explored by discarding transitions bounding these clocks. Notice that discarding edges may split the SCC into smaller SCCs. The difficult task is to discover the smaller SCCs and explore them on-the-fly.

The second challenge is to handle SCCs with zero-checks, that is clocks that are tested for value being zero. In this case, the zone graph does not contain enough information to detect time progress. This is where the guessing zone graph construction is required. The involving part of the problem is to introduce this construction on-the-fly. In particular the part of the guessing zone graph that is explored should be restricted only to the transitions from the SCC containing zero-checks.

We propose an algorithm that fulfills both challenges. In the worst case, it runs in time $\mathcal{O}\left(|Z G| \cdot(|X|+1)^{2}\right)$. When the automaton does not have zero checks it runs in time $\mathcal{O}(|Z G| \cdot(|X|+1))$. When the automaton further has no blocking clocks, it runs in time $\mathcal{O}(|Z G|)$. Our algorithm further incorporates an improvement that proves powerful in practice. Indeed, non-Zenoness could be 
established from situations where the value of some clock is known to increase by 1 infinitely often. Even in the presence of zero-checks, this property avoids exploring the guessing zone graph. Again, this improvement is applied on-the-fly.

Next, we show that all these operations on SCCs can be handled efficiently as the memory needed by our algorithm is comparable to the memory usage of the strongly non-Zeno approach in [16].

Finally, we also report some successful experiments of our algorithm on examples from the literature. All the experiments that we have conducted show that non-Zenoness constructions need not be applied mechanically, hence validating our approach.

Related work. Timed automata with Büchi conditions have been introduced in the seminal paper [1]. The zone approach to model-checking has been introduced in [5] and later used in the context of timed automata [11]. The Büchi emptiness problem using zones has been studied in $[6,16,15]$. The idea of the strongly non-Zeno approach first appears in [14]. The strongly non-Zeno construction has been implemented in the tool Profounder [16]. Finally, the recent paper [12] introduces the guessing zone graph construction.

Outline. The paper is organized as follows. In Section 2 we define the emptiness problem. Then we present the two approaches that ensure time progress in Section 3. We detail our algorithm and its optimizations in Section 4. Finally, we discuss experiments and future work in Section 5.

\section{The Emptiness Problem for Timed Büchi Automata}

\subsection{Timed Büchi Automata}

Let $X$ be a set of clocks, i.e., variables that range over $\mathbb{R}_{\geq 0}$, the set of nonnegative real numbers. Clock constraints are conjunctions of comparisons of variables with integer constants, e.g. $(x \leq 3 \wedge y>0)$. Let $\Phi(X)$ denote the set of clock constraints over clock variables $X$.

A clock valuation over $X$ is a function $\nu: X \rightarrow \mathbb{R}_{\geq 0}$. We denote $\mathbb{R}_{>0}^{X}$ the set of clock valuations over $X$, and $\mathbf{0}: X \rightarrow\{0\}$ the valuation that associates 0 to every clock in $X$. We write $\nu \models \phi$ when $\nu$ satisfies $\phi$, i.e. when every constraint in $\phi$ holds after replacing every $x$ by $\nu(x)$.

For a valuation $\nu$ and $\delta \in \mathbb{R}_{\geq 0}$, let $(\nu+\delta)$ be the valuation such that $(\nu+$ $\delta)(x)=\nu(x)+\delta$ for all $x \in X$. For a set $R \subseteq X$, let $[R] \nu$ be the valuation such that $([R] \nu)(x)=0$ if $x \in R$ and $([R] \nu)(x)=\nu(x)$ otherwise.

A Timed Büchi Automaton (TBA) is a tuple $\mathcal{A}=\left(Q, q_{0}, X, T, A c c\right)$ where $Q$ is a finite set of states, $q_{0} \in Q$ is the initial state, $X$ is a finite set of clocks, $A c c \subseteq Q$ is a set of accepting states, and $T \subseteq Q \times \Phi(X) \times 2^{X} \times Q$ is a finite set of transitions $\left(q, g, R, q^{\prime}\right)$ where $g$ is a guard, and $R$ is a reset of the transition. Examples of TBA are depicted in Figure 1.

A configuration of $\mathcal{A}$ is a pair $(q, \nu) \in Q \times \mathbb{R}_{\geq 0}^{X}$; with $\left(q_{0}, \mathbf{0}\right)$ being the initial configuration. A discrete transition between configurations $(q, \nu) \stackrel{t}{\rightarrow}\left(q^{\prime}, \nu^{\prime}\right)$ for 
$t=\left(q, g, R, q^{\prime}\right)$ is defined when $\nu \vDash g$ and $\nu^{\prime}=[R] \nu$. We also have delay transitions between configurations: $(q, \nu) \stackrel{\delta}{\rightarrow}(q, \nu+\delta)$ for every $q, \nu$ and $\delta \in \mathbb{R}_{\geq 0}$. We write $(q, \nu) \stackrel{\delta, t}{\longrightarrow}\left(q^{\prime}, \nu^{\prime}\right)$ if $(q, \nu) \stackrel{\delta}{\rightarrow}(q, \nu+\delta) \stackrel{t}{\longrightarrow}\left(q^{\prime}, \nu^{\prime}\right)$.

A run of $\mathcal{A}$ is a finite or infinite sequence of configurations connected by $\stackrel{\delta, t}{\longrightarrow}$ transitions, starting from the initial state $q_{0}$ and the initial valuation $\nu_{0}=\mathbf{0}$ :

$$
\left(q_{0}, \nu_{0}\right) \stackrel{\delta_{0}, t_{0}}{\longrightarrow}\left(q_{1}, \nu_{1}\right) \stackrel{\delta_{1}, t_{1}}{\longrightarrow} \cdots
$$

A run $\sigma$ satisfies the Büchi condition if it visits accepting configurations infinitely often, that is configurations with a state from Acc. A duration of the run is the accumulated delay: $\sum_{i \geq 0} \delta_{i}$. A run $\sigma$ is Zeno if its duration is bounded.

Definition 1. The Büchi non-emptiness problem is to decide if $\mathcal{A}$ has a nonZeno run satisfying the Büchi condition.

The class of TBA we consider is usually known as diagonal-free TBA since clock comparisons like $x-y \leq 1$ are disallowed. Since we are interested in the Büchi non-emptiness problem, we can consider automata without an input alphabet and without invariants since they can be simulated by guards.

The Büchi non-emptiness problem is known to be PSPACE-complete [1]

\subsection{The Zone Graph}

A zone is a set of valuations defined by a conjunction of two kinds of constraints: comparison of the difference between two clocks with a constant, or comparison of the value of a single clock with a constant. For instance $(x-y \geq 1) \wedge(y<2)$ is a zone.

The transition relation on valuations can be transposed to zones. Let $\vec{Z}$ be a zone denoting the result of time elapse from $Z$, that is, the set of all valuations $\nu^{\prime}$ such that $\nu^{\prime}=\nu+\delta$ for some $\nu \in Z$ and $\delta \in \mathbb{R}_{\geq 0}$. We have $(q, Z) \stackrel{t}{\rightarrow}\left(q^{\prime}, Z^{\prime}\right)$ if $Z^{\prime}$ is the set of valuations $\nu^{\prime}$ such that $(q, \nu) \stackrel{t}{\rightarrow}\left(q^{\prime}, \nu^{\prime}\right)$ for some $\nu \in \vec{Z}$. It can be checked that $Z^{\prime}$ is a zone. Difference Bound Matrices (DBMs) can be used for the representation of zones [11]. Transitions are computed efficiently for zones represented by DBMs.

However, the number of reachable symbolic configurations $(q, Z)$ may not be finite [10]. Hence tools like Kronos or Uppaal use an approximation operator Approx that reduces the set of zones to a finite set. The zone graph of $\mathcal{A}$, denoted $Z G(\mathcal{A})$, has nodes of the form $(q, A \operatorname{pprox}(Z))$. Observe that $Z G(\mathcal{A})$ only has finitely many nodes. The initial node is $\left(q_{0}, Z_{0}\right)$ where $q_{0}$ is the initial state of $\mathcal{A}$ and $Z_{0}$ is the zone where all the clocks are equal to zero. The transitions of the zone graph are $(q, Z) \stackrel{t}{\rightarrow}\left(q^{\prime}\right.$, Approx $\left.\left(Z^{\prime}\right)\right)$ instead of $(q, Z) \stackrel{t}{\rightarrow}\left(q^{\prime}, Z^{\prime}\right)$.

It remains to define Approx in such a way that verification results for $Z G(\mathcal{A})$ entail similar results for $\mathcal{A}$. Let $M$ be the maximal constant in $\mathcal{A}$. $M$ defines the precision of the approximation. We define the region equivalence over valuations as $\nu \sim_{M} \nu^{\prime}$ iff for every $x, y \in X$ : 
1. $\nu(x)>M$ iff $\nu^{\prime}(x)>M$;

2. if $\nu(x) \leq M$, then $\lfloor\nu(x)\rfloor=\left\lfloor\nu^{\prime}(x)\right\rfloor$;

3. if $\nu(x) \leq M$, then $\{\nu(x)\}=0$ iff $\left\{\nu^{\prime}(x)\right\}=0$;

4. for every integer $c \in(-M, M): \nu(x)-\nu(y) \leq c$ iff $\nu^{\prime}(x)-\nu^{\prime}(y) \leq c$.

The first three conditions ensure that $\nu$ and $\nu^{\prime}$ satisfy the same guards. The last one enforces that for every $\delta \in \mathbb{R}_{\geq 0}$ there is $\delta^{\prime} \in \mathbb{R}_{\geq 0}$, such that valuations $\nu+\delta$ and $\nu^{\prime}+\delta^{\prime}$ satisfy the same guards. This definition of region equivalence is introduced in [7]. Notice that it is finer than the equivalence defined in [1] thanks to the last condition which is needed for correctness of Theorem 1 below.

An equivalence class of $\sim_{M}$ is called a region. For a zone $Z$, we define $A \operatorname{pprox}(Z)$ as the smallest union of regions that is convex and contains $Z$. Notice that there are finitely many regions, hence finitely many approximated zones.

We call a path in the zone graph a finite or infinite sequence of transitions:

$$
\left(q_{0}, Z_{0}\right) \stackrel{t_{0}}{\longrightarrow}\left(q_{1}, Z_{1}\right) \stackrel{t_{1}}{\rightarrow} \cdots
$$

A path is initialized when it starts from the initial node $\left(q_{0}, Z_{0}\right)$. A run of $\mathcal{A}$ $\left(q_{0}, \nu_{0}\right) \stackrel{\delta_{0}, t_{0}}{\longrightarrow}\left(q_{1}, \nu_{1}\right) \stackrel{\delta_{1}, t_{1}}{\longrightarrow} \cdots$ is an instance of a path if $\nu_{i} \in Z_{i}$ for every $i \geq 0$. The path is called an abstraction of the run.

Approx preserves many verification properties, and among them Büchi accepting paths as stated in the following theorem.

Theorem 1 ([15]). Every path in $Z G(\mathcal{A})$ is an abstraction of a run of $\mathcal{A}$, and conversely, every run of $\mathcal{A}$ is an instance of a path in $Z G(\mathcal{A})$.

As a consequence, every run of $\mathcal{A}$ that satisfies the accepting conditions yields a path in $Z G(\mathcal{A})$ that also satisfies the accepting conditions, and conversely. However, it is not possible to determine from a path in $Z G(\mathcal{A})$ if it can be instantiated to a non-Zeno run of $\mathcal{A}$. Hence, we need to preserve more information either in the automaton or in the zone graph.

\section{Solutions to the Zenoness Problem}

Theorem 1 is a first step towards an algorithm for the Büchi emptiness problem working from the zone graph. It remains to ensure that a given path in $Z G(\mathcal{A})$ has a non-Zeno instance in $\mathcal{A}$. Observe that the zone graph is not pre-stable: a transition $(q, Z) \stackrel{t}{\rightarrow}\left(q^{\prime}, Z^{\prime}\right)$ may not have an instance for a valuation $\nu \in Z$. Hence zones are not precise enough to detect time progress.

For example, the state 3 of $\mathcal{A}_{1}$ in Figure 1 is reachable with zone $Z=(0 \leq$ $x \wedge x \leq y)$ on path from 0 via 2 . At first sight, it seems that time can elapse in configuration $(3, Z)$. This is not the case as the transition from 3 to 0 forces $x$ to be equal to 0 . Hence, $Z$ has to be separated in two zones: $x=0 \wedge x \leq y$ that can take the transition from 3 to 0 , and $x>0 \wedge x \leq y$ that is a deadlock node.

We present two approaches from the literature to overcome this problem. Both are used in our algorithm in section 4 . They rely on the properties of the 


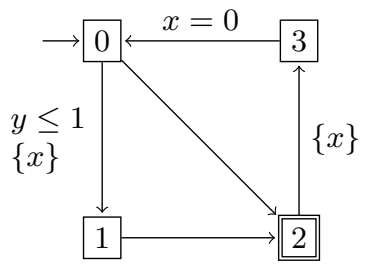

$\mathcal{A}_{1}$

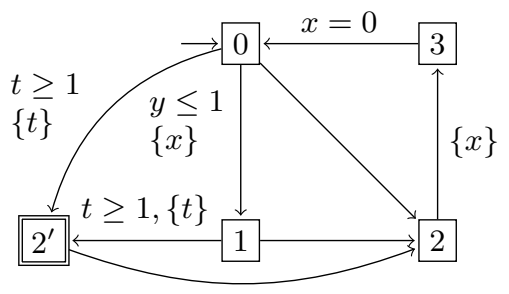

$\operatorname{SNZ}\left(\mathcal{A}_{1}\right)$

Fig. 1. A Timed Automaton and a Strongly non-Zeno Timed Automaton.

transitions $(q, Z) \stackrel{g ; R}{\longrightarrow}\left(q^{\prime}, Z^{\prime}\right)$ in $Z G(\mathcal{A})$. We say that a transition bounds $x$ from above if there exists $c \geq 1$ such that $\vec{Z} \wedge g \Rightarrow(x \leq c)$. Observe that this is equivalent to $\nu^{\prime \prime}(x) \leq c$ for every valuation $\nu^{\prime \prime}$ such that both $(q, \nu) \stackrel{\delta}{\rightarrow}\left(q, \nu^{\prime \prime}\right)$ and $\nu^{\prime \prime} \models g$. Similarly a transition bounds $x$ from below when $\vec{Z} \wedge g \Rightarrow(x \geq c)$; it zero checks $x$ when $\vec{Z} \wedge g \Rightarrow(x=0)$. Finally, a transition resets $x$ when $x$ belongs to $R$. Notice that all these properties are easily checked using DBMs for zones and guards.

\subsection{Adding One Extra Clock}

A common solution to deal with Zeno runs is to transform a TBA into a strongly non-Zeno automaton, i.e. such that all runs satisfying the Büchi condition are guaranteed to be non-Zeno.

The construction in $[16,2]$ adds one clock $t$ and duplicates the accepting states in order to ensure that at least one unit of time has passed between two visits to an accepting state. Figure 1 presents $\mathcal{A}_{1}$ along with its strongly non-Zeno counterpart $S N Z\left(\mathcal{A}_{1}\right)$. The correctness of the approach comes from Lemma 1 below, the proof of which is omitted.

Lemma 1. If a path in $Z G(\mathcal{A})$ visits infinitely often both a transition that bounds some clock $x$ from below and a transition that resets the same clock $x$, then all its instances are non-Zeno.

The converse also holds: if $\mathcal{A}$ has a non-Zeno accepting run, then 1 time unit elapses infinitely often. Hence, the guard $t \geq 1$ is satisfied infinitely often and there exists an accepting run of $S N Z(\mathcal{A})$. By Theorem 1 one can find an accepting path in $Z G(S N Z(\mathcal{A}))$.

Despite being simple, this construction may lead to an exponential blowup. It is shown in [12] that there exists some TBA $\mathcal{A}$ such that the zone graph of $S N Z(\mathcal{A})$ has size $|Z G(\mathcal{A})| .2^{\mathcal{O}(|X|)}$. Our algorithm described in section 4 will use a similar idea as an optimization. However, no new clocks are added and no blowup is inflicted. 


\subsection{The Guessing Zone Graph}

Another solution was introduced in [12] in order to avoid the exponential blowup. Consider a path in $Z G(\mathcal{A})$ that only has Zeno instances. Two situations may occur. Either the path has infinitely many transitions that bound some clock $x$ from above, but only finitely many transitions that reset $x$. Then, the total time elapsed is bounded. Or, time cannot elapse at all because of zero-checks, as this is the case in state 3 of $\mathcal{A}_{1}$ in Figure 1 .

Zero-checks are handled thanks to a modification of the zone graph. The nodes will now be triples $(q, Z, Y)$ where $Y \subseteq X$ is the set of clocks that can potentially be checked to 0 . It means in particular that other clock variables, i.e. those from $X-Y$ are assumed to be bigger than 0 . We write $(X-Y)>0$ for the constraint saying that all the variables in $X-Y$ are not 0 .

Definition 2. Let $\mathcal{A}$ be a TBA over a set of clocks $X$. The guessing zone graph $G Z G(\mathcal{A})$ has nodes of the form $(q, Z, Y)$ where $(q, Z)$ is a node in $Z G(\mathcal{A})$ and $Y \subseteq X$. The initial node is $\left(q_{0}, Z_{0}, X\right)$, with $\left(q_{0}, Z_{0}\right)$ the initial node of $Z G(\mathcal{A})$. There is a transition $(q, Z, Y) \stackrel{t}{\rightarrow}\left(q^{\prime}, Z^{\prime}, Y \cup R\right)$ in $G Z G(\mathcal{A})$ if there is a transition $(q, Z) \stackrel{t}{\rightarrow}\left(q^{\prime}, Z^{\prime}\right)$ in $Z G(\mathcal{A})$ with $t=\left(q, g, R, q^{\prime}\right)$, and there are valuations $\nu \in Z$, $\nu^{\prime} \in Z^{\prime}$, and $\delta$ such that $\nu+\delta \vDash(X-Y)>0$ and $(q, \nu) \stackrel{\delta, t}{\longrightarrow}\left(q, \nu^{\prime}\right)$. We also introduce a new auxiliary letter $\tau$, and put transitions $(q, Z, Y) \stackrel{\tau}{\rightarrow}\left(q, Z, Y^{\prime}\right)$ for $Y^{\prime}=\emptyset$ or $Y^{\prime}=Y$.

Observe that the definition of transitions reflects the intuition about $Y$ we have described above. Indeed, the additional requirement on the transition $(q, Z, Y) \stackrel{t}{\rightarrow}\left(q^{\prime}, Z^{\prime}, Y \cup R\right)$ is that it should be realizable when the clocks outside $Y$ are strictly positive; so there should be a valuation satisfying $(X-Y)>0$ that realizes this transition. This construction entails that from a node $(q, Z, \emptyset)$ every reachable zero-check is preceded by the reset of the variable that is checked, and hence nothing prevents a time elapse in this node. We call such a node clear. We call a node $(q, Z, Y)$ accepting if it contains an accepting state $q$. Figure 4 depicts the part of $G Z G\left(\mathcal{A}_{1}\right)$ reachable from node $(3, x==0,\{x, y\})$ with state 1 and all its transitions removed from $\mathcal{A}_{1}$.

Notice that directly from Definition 2 it follows that a path in $G Z G(\mathcal{A})$ determines a path in $Z G(\mathcal{A})$ obtained by removing $\tau$ transitions and the third component from nodes.

We say that a path is blocked if there is a variable that is bounded from above by infinitely many transitions but reset by only finitely many transitions on the path. Otherwise the path is called unblocked. We have the following result.

Theorem 2 ([12]). A TBA $\mathcal{A}$ has a non-Zeno run satisfying the Büchi condition iff there exists an unblocked path in $G Z G(\mathcal{A})$ visiting both an accepting node and a clear node infinitely often.

It is shown in the same paper that the proposed solution does not produce an exponential blowup. This is due to the fact that the zones reachable in $Z G(\mathcal{A})$ 
order the clocks. More precisely, if $Z$ is the zone of a reachable node in $Z G(\mathcal{A})$ then for every two clocks $x, y, Z$ implies that at least one of $x \leq y$, or $y \leq x$ holds [12]. Now, it can be checked that for every node $(q, Z, Y)$ reachable in $G Z G(\mathcal{A})$, the set $Y$ respects the order given by $Z$, that is whenever $y \in Y$ and $Z$ implies $x \leq y$ then $x \in Y$. In the end, a nice polynomial bound is obtained on the size of the guessing zone graph.

Lemma 2 ([12]). Let $|Z G(\mathcal{A})|$ be the size of the zone graph, and $|X|$ be the number of clocks in $\mathcal{A}$. The number of reachable nodes of $G Z G(\mathcal{A})$ is bounded by $|Z G(\mathcal{A})| \cdot(|X|+1)$.

\section{Solving the emptiness problem on-the-fly}

\subsection{On-the-fly emptiness check on the guessing zone graph}

We propose an on-the-fly algorithm for the Büchi emptiness problem based on Theorem 2. It requires to find an unblocked path in $G Z G(\mathcal{A})$ that visits both an accepting node and a clear node infinitely often. To make the presentation of the algorithm simpler, we assume that there is one distinguished Büchi condition that labels exactly all the clear nodes. Hence we search for an unblocked path in $G Z G(\mathcal{A})$ that satisfies the Büchi conditions.

Observe that we do not need to exhibit the path; proving its existence is simpler and sufficient ${ }^{1}$. Our problem can thus be stated over the SCCs of $G Z G(\mathcal{A})$. A clock is blocking in an SCC if it is not reset by any transition in the SCC, but it is bounded from above by one of them. An SCC is unblocked when it has no blocking clock. Hence, finding an SCC that is unblocked and that contains an accepting node (for every Büchi condition) yields the required path. Conversely, from such a path, we have a satisfactory SCC.

The algorithm essentially involves three tasks: (1) detecting an accepting SCC, (2) inferring any blocking clocks in the SCC, and (3) re-exploring the SCC with blocking transitions removed. Since the blocking transitions might involve resets of other clocks, the SCCs obtained after removing blocking transitions might in turn contain new blocking clocks. These tasks have to be accomplished on-the-fly. The Couvreur's algorithm [9] finds accepting SCCs in (untimed) Büchi automata on-the-fly. We first adapt it to infer blocking clocks. Next we propose a modification to enable performing the third task. This last contribution is particularly involving. Our algorithm is depicted in Figure 2.

Couvreur's algorithm The Couvreur's algorithm, can be viewed as three functions check_dfs, merge_scc and close_scc. The algorithm annotates every node with an integer $d f$ snum and a boolean opened. The variable $d f$ snum is assigned based on the order of appearance of the nodes during the depth-first

\footnotetext{
${ }^{1}$ The path is a counter-example to the emptiness of the automaton, hence it can be an expected outcome of the algorithm. ¿s in the untimed case, a short counter-example can be generated in a separate task from the outcome of our algorithm.
} 
search. The opened bit is set to true when the node is just opened for exploration by check_dfs and is set to false by close_scc when the entire SCC of the node has been completely explored. The algorithm uses two stacks Roots and Active. The Roots stack stores the root of each SCC in the current search path. The root is the node of the SCC that was first visited by the DFS. If the roots stack is $s_{0} s_{1} \ldots s_{n}$, then for $0 \leq i \leq n-1, s_{i}$ is the root of the SCC containing all the nodes with $d f$ snum between $s_{i} . d f$ snum and $s_{i+1}$.df snum that have opened set to true and $s_{n}$ is the root of the SCC containing all nodes with $d f$ snum greater than $s_{n}$.df snum which have opened set to true.

The main function $c h e c k \_d f s$ proceeds by exploring the graph in depth-first search (DFS) order. When a successor $t$ of the current node $s$ is found, $t$.df snum being zero implies $t$ has not been visited yet and $t$.opened being true implies that $t$ belongs to the same SCC as $s$. When $t$ belongs to the SCC of $s$, all the nodes visited in the path from $t$ to $s$ also belong to the SCC of $s$. These nodes are collected by the function merge_scc, which finds the root $s_{i}$ of $t$ and repeatedly pops the Roots stack so that $s_{i}$ comes to the top, signifying that it is the root of the SCC containing all the nodes visited from $t$ to $s$. A maximal SCC is detected when all the transitions of the current node $s$ have been explored, with $s$ being on the top of the Roots stack. The close_scc function is now called that sets the opened bit of all nodes in the SCC rooted at $s$ to false. To identify these nodes, the Active stack is used, which stores all the nodes of the partially explored SCCs, in the order of the $d f$ snum.

Detecting blocking clocks Observe that each node $s$ in the Roots stack represents an SCC rooted at $s$. The idea is to augment the Roots stack so that along with each node $s$, the set of clocks $(u b)$ that are bounded above and the set of clocks $r$ that are reset in a transition of the SCC rooted at $s$, are also stored. The set $u b-r$ gives the set of blocking clocks of the SCC rooted at $s$. To achieve this, SCCs are stored in the Roots stack as tuples $\left(s, a, u b, r, u b_{i n}, r_{i n}\right)$ where the extra sets $u b_{i n}$ and $r_{i n}$ store respectively the clocks that are bounded above and reset in the transition leading to $s$. The second modification occurs in merge_scc which now accumulates these sets while popping the nodes from the Roots stack. Figure 3 gives a schematic diagram of the merging procedure.

Re-exploring blocked SCCs When a maximal SCC $\Gamma$ is detected, the set $u b-r$ of the root gives the set of blocking clocks. If $u b-r$ is not empty, $\Gamma$ needs to be re-explored with the transitions bounding clocks of $b l k \cup(u b-r)$ removed. Here $b l k$ denotes the blocked clocks when $\Gamma$ was being explored. Doing this would split $\Gamma$ into some $p$ smaller maximal SCCs $\Gamma_{1}, \ldots, \Gamma_{p}$ such that $\Gamma=\bigcup_{i=1}^{i=p} \Gamma_{i}$. Each $\Gamma_{i}$ is reachable from the root of $\Gamma$. Thus, $G Z G(\mathcal{A})$ would have an accepting run if some $\Gamma_{i}$ contains an unblocked accepting cycle. The objective is to identify and explore the sub SCCs $\Gamma_{1}, \ldots, \Gamma_{p}$ on-the-fly. The function close_scc is modified to enable on-the-fly re-exploration with blocked clocks.

Firstly, an additional bit per node called explore is needed to identify the nodes that have to be explored again. When $\Gamma$ is detected and close_scc called, 


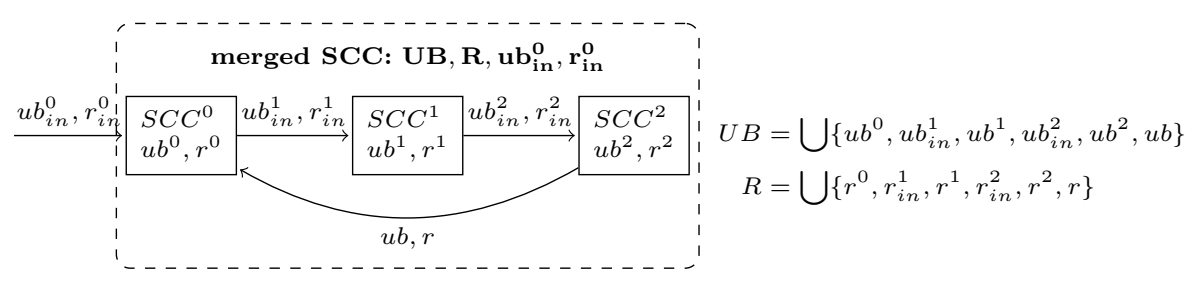

Fig. 3. How $u b$ sets and $r$ sets are merged.

\subsection{On-the-fly emptiness check on the zone graph}

As stated in section 3.2, the guessing zone graph construction detects nodes where time elapse is not prohibited by future zero checks. However, in the absence of zero checks, this construction is not necessary. Recall that $G Z G(\mathcal{A})$ has $|X|+1$ times more nodes than $Z G(\mathcal{A})$. Therefore, it is sufficient to consider the guessing zone graph construction only on maximal SCCs with zero checks. We propose an on-the-fly algorithm that checks for an accepting and unblocked SCC on the zone graph $Z G(\mathcal{A})$, detects zero checks and adopts the emptiness check algorithm on the guessing zone graph only when required. The algorithm shown in Figure 2 is run on $Z G(\mathcal{A})$ instead of $G Z G(\mathcal{A})$. When a maximal SCC $\Gamma$ is detected in line 19 , it is required to know if $\Gamma$ contains any zero checks.

Detecting zero checks This is similar to the detection of blocking clocks. Two extra bits $z c$ and $z c_{i n}$ are stored along with every tuple $(s, \ldots)$ in the Roots stack. $z c_{i n}$ tracks zero checks in the transition leading to $s$ and $z c$ remembers if there is a zero check in the SCC rooted at $s$. When an SCC is detected, the information is merged in merge_scc in a way similar to the schematic diagram shown in Figure 3. Thus when $\Gamma$ is detected, the $z c$ bit of the root reveals the presence of zero checks in $\Gamma$. The following lemma says that the algorithm can be terminated if $\Gamma$ is accepting, unblocked and free from zero-checks.

Lemma 3. If a reachable $S C C$ in $Z G(\mathcal{A})$ is accepting, unblocked and free from zero-checks, then $\mathcal{A}$ has a non-Zeno accepting run.

The interesting case occurs when $\Gamma$ does have zero checks. In this case, we apply the guessing zone graph construction only to the nodes of $\Gamma$. If $\left(q^{\Gamma}, Z^{\Gamma}\right)$ is the root of $\Gamma$, the guessing zone graph is constructed on-the-fly starting from $\left(q^{\Gamma}, Z^{\Gamma}, X\right)$. Intuitively, we assume that the set of clocks $X$ could potentially be zero at this node and start the exploration. We say that a run $\rho$ of $\mathcal{A}$ ends in a maximal SCC $\Gamma$ of $Z G(\mathcal{A})$ if a suffix of $\rho$ is an instance of a path in $\Gamma$. Let $G Z G(\mathcal{A})_{\mid \Gamma}$ be the part of $G Z G(\mathcal{A})$ restricted to nodes and transitions that occur in $\Gamma$.

Lemma 4. Let $\Gamma$ be a reachable maximal $S C C$ in $Z G(\mathcal{A})$, with $\operatorname{root}\left(q^{\Gamma}, Z^{\Gamma}\right)$. $\mathcal{A}$ has an accepting non-Zeno run ending in $\Gamma$ iff $G Z G(\mathcal{A})_{\mid \Gamma}$ has an unblocked accepting $S C C$ reachable from $\left(q^{\Gamma}, Z^{\Gamma}, X\right)$. 
Handling the zero checks The function $c l o s e \_s c c$ is modified to identify any zero-checks in the current maximal SCC $\Gamma$ that was detected. If $\Gamma$ contains zero checks, then check $d f s$ is called with the node $\left(q^{\Gamma}, Z^{\Gamma}, X\right)$ and the current set $b l k$ of blocking clocks. Note that the explore bit is true for all the nodes in $\Gamma$. Each time a new node $t=(q, Z, Y)$ of $G Z G(\mathcal{A})$ is discovered in the for loop $s \stackrel{g ; r}{\longrightarrow} t$, it is explored only when $(q, Z)$.explore is true. When the exploration of $G Z G(\mathcal{A})$ terminates, the explore bit of all the nodes of $\Gamma$ are set to false. The explore bit is thus responsible for the restriction of the search on $G Z G(\mathcal{A})$ only to the nodes that occur in $\Gamma$. In particular, this happens on-the-fly.

\subsection{An optimization}

We propose an optimization to the algorithm to enable quicker termination in some cases. We take advantage of clocks in an SCC that are bounded from below, that is of the form $x>c$ or $x \geq c$ with $c \geq 1$. We make use of Lemma 1 which says that if an SCC $\Gamma$ contains a transition that bounds a clock from below and a transition that resets the same clock, then any path of $\Gamma$ including both these transitions should have non-Zeno instantiations. This is irrespective of whether $\Gamma$ contains zero checks. Notice that no new clock is added.

To implement the above optimization, we add clock sets $l b$ and $l b_{\text {in }}$ to the Roots stack entries. The set $l b$ keeps track of the clocks that are bounded below in the SCC. $l b$ and $l b_{i n}$ are handled as explained in Figure 3 for the sets $u b$ and $u b_{i n}$. We also modify the condition for $L(\mathcal{A})$ to be empty. We conclude as soon as an SCC, not necessarily maximal, is accepting and it bounds below and resets the same clock. Observe that it is not useful to keep track of the clocks that are bounded from below while solving zero-checks on $G Z G(\mathcal{A})$ as any transition that sets a lower bound in $G Z G(\mathcal{A})$ has already been visited in $Z G(\mathcal{A})$.

\subsection{The Global Algorithm and Implementation Issues}

A run of our algorithm is illustrated in Figure 4. Exploring $Z G\left(\mathcal{A}_{1}\right)$, it computes the maximal SCC rooted at node 2 that is blocked by $y$. Hence, it re-explores that SCC with set of blocking clocks $b l k=\{y\}$. This is seen from the double edges. Notice that the edge from node 5 to node 2 is not crossed as it is discarded by $b l k$. The algorithm now computes the SCC $\Gamma$ that contains the nodes 4, 5 and 6 , that is maximal w.r.t. blk. $\Gamma$ is unblocked and accepting, but it has zero-checks. Hence the algorithm explores $G Z G\left(\mathcal{A}_{1}\right)$ starting from node $(3, x==0,\{x, y\})$. It does not explore any transition outside of $\Gamma$. It eventually finds a non-maximal $\mathrm{SCC}$ in $G Z G\left(\mathcal{A}_{1}\right)$ that proves $L\left(\mathcal{A}_{1}\right) \neq \emptyset$.

Finally, we discuss memory issues for the implementation of our algorithm. Information about the SCCs in $Z G(\mathcal{A})$ is stored in the Roots stack as tuples $\left(s, a, l b, u b, r, z c, l b_{i n}, u b_{i n}, r_{i n}, z c_{i n}\right)$. An amount of $6 .|X|+2$ bits of memory is required to store the membership of each clock to the 6 sets, and the zero-check information. In the worst case, all the nodes in $Z G(\mathcal{A})$ have an entry in the Roots stack. Similarly, SCCs in $G Z G(\mathcal{A})$ are stored as tuples $\left(s, a, u b, r, u b_{i n}, r_{i n}\right)$ in 


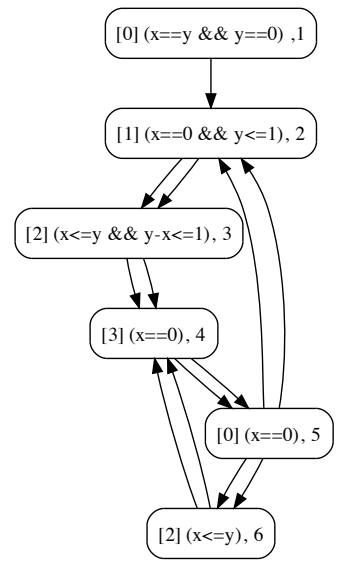

$\mathbf{Z G}\left(\mathcal{A}_{\mathbf{1}}\right)$

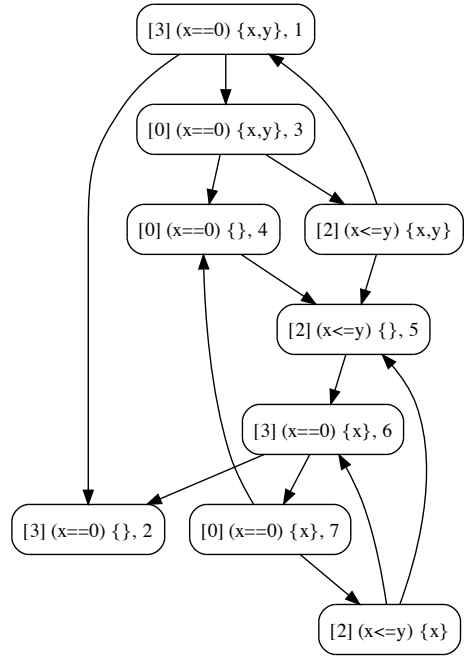

Part of $\mathbf{G Z G}\left(\mathcal{A}_{\mathbf{1}}\right)$

Fig. 4. Zone Graphs of $\mathcal{A}_{1}$.

the Roots stack. 4. $|X|$ bits allow to represent the content of those 4 sets. We estimate than the overhead due to our algorithm is $4 .|X| \cdot(|X|+1)$ due to the size of $G Z G(\mathcal{A})$ w.r.t. the size of $Z G(\mathcal{A})$.

We eventually compare to the strongly non-Zeno construction. Running the Couvreur's algorithm requires to store smaller tuples $(s, a)$. However, the zone in $s$ is represented by a DBM which is an $|X|+1$-square matrix containing 32 bits integers. Hence, adding one clock increases the memory footprint by $(2 .|X|+3) .32$ bits. Recall furthermore than $Z G(S N Z(\mathcal{A}))$ can be $2^{\mathcal{O}(|X|)}$ bigger than $Z G(\mathcal{A})$. In the worst case all these nodes are on the Roots stack.

\section{Experiments and Conclusion}

We have implemented our algorithms in a prototype verification tool. The table below presents the results that we obtained on several classical examples. The models are products of Timed Büchi Automata that encode both the processes in the system and the property to verify. The "Zone Graph" column gives the number of nodes in the zone graph. Next, for the "Strongly non-Zeno" construction, we give the size of the resulting zone graph followed by the number of nodes that are visited during verification; similarly for the "Guessing Zone Graph" where the last column corresponds to our fully optimized algorithm.

We have considered three types of properties: reachability properties (mutual exclusion, collision detection for CSMA/CD), liveness properties (access to the resource infinitely often), and bounded response properties (which are reachability properties with real-time requirements). When checking reachability properties we would like counter examples to be feasible by real systems. 
This is not the case with Zeno counter examples. Hence Timed Büchi Automata offer a nice framework as they discard Zeno runs.

The strongly non-Zeno construction outperforms the guessing zone graph construction for reachability properties. This is particularly the case for mutual exclusion on the Fischer's protocol and collision detection for the CSMA/CD protocol. For liveness properties, the results are mitigated. On the one hand, the strongly non-Zeno property is once again more efficient for the CSMA/CD protocol. On the other hand the differences are tight in the case of Fischer protocol. The guessing zone graph construction distinguishes itself for bounded response properties. Indeed, the Train-Gate model is an example of exponential blowup for the strongly non-Zeno construction.

We notice that on-the-fly algorithms perform well. Even when the graphs are big, particularly in case when automata are not empty, the algorithms are able to conclude after having explored only a small part of the graph. Our optimized algorithm outperforms the two others on most examples. Particularly, for the CSMA/CD protocol with 5 stations our algorithm needs to visit only 4841 nodes while the two other methods visited 8437 and 21038 nodes. This confirms our initial hypothesis: most of the time, the zone graph contains enough information to ensure time progress.

Our last optimization also proves useful for the FDDI protocol example. One of its processes has zero checks, but since some other clock is bounded from below and reset, it was not necessary to explore the guessing zone graph to conclude non-emptiness.

\begin{tabular}{|l|r||r|r||r|r|r|}
\hline \multirow{2}{*}{ Models } & \multicolumn{2}{|l|}{ Zone Graph } & Strongly non-Zeno & \multicolumn{2}{|c|}{ Guessing Zone Graph } \\
\cline { 2 - 6 } & size & size & visited & size & visited & visited opt. \\
\hline Train-Gate2 (mutex) & 134 & 194 & 194 & 400 & 400 & 134 \\
Train-Gate2 (bound. resp.) & 988 & 227482 & 352 & 3840 & 1137 & 292 \\
Train-Gate2 (liveness) & 100 & 217 & 35 & 298 & 53 & 33 \\
\hline Fischer3 (mutex) & 1837 & 3859 & 3859 & 7292 & 7292 & 1837 \\
Fischer4 (mutex) & 46129 & 96913 & 96913 & 229058 & 229058 & 46129 \\
Fischer3 (liveness) & 1315 & 4962 & 52 & 5222 & 64 & 40 \\
Fischer4 (liveness) & 33577 & 147167 & 223 & 166778 & 331 & 207 \\
\hline FDDI3 (liveness) & 508 & 1305 & 44 & 3654 & 79 & 42 \\
FDDI5 (liveness) & 6006 & 15030 & 90 & 67819 & 169 & 88 \\
FDDI3 (bound. resp.) & 6252 & 41746 & 59 & 52242 & 114 & 60 \\
\hline CSMA/CD4 (collision) & 4253 & 7588 & 7588 & 20146 & 20146 & 4253 \\
CSMA/CD5 (collision) & 45527 & 80776 & 80776 & 260026 & 260026 & 45527 \\
CSMA/CD4 (liveness) & 3038 & 9576 & 1480 & 14388 & 3075 & 832 \\
CSMA/CD5 (liveness) & 32751 & 120166 & 8437 & 186744 & 21038 & 4841 \\
\hline
\end{tabular}

Conclusion and Future Work. In this paper, we have presented an algorithm for the emptiness problem of Timed Büchi Automata. We claim that our algorithm is on-the-fly as (1) it computes the zone graph during the emptiness check; (2) it does not store the graph, in particular when it splits blocked SCCs; and (3) it stops as soon as an SCC that contains a non-Zeno accepting run is found. Our algorithm is inspired from the Couvreur's algorithm. However, the handling of bounded clocks and the application of the guessing zone graph construction on-the-fly, are two substantial increments. The previous examples are academic case studies; still they show that Büchi properties can be checked as efficiently as reachability properties for Timed Automata, despite the non-Zenoness requirement. 
As a future work we plan to extend our algorithm to commonly used syntactic extensions of Timed Automata. For instance Uppaal and Kronos allow to reset clocks to arbitrary values, which is convenient for modeling real life systems. This would require to extend the guessing zone graph construction, and consequently our algorithm. Another interesting question is to precisely characterize the situations where an exponential blowup occurs in the strongly non-Zeno construction. To conclude, it can be seen that the ideas of the prototype could be used to construct a full-fledged tool like Profounder, which implements the strongly non-Zeno construction.

\section{References}

1. R. Alur and D.L. Dill. A theory of timed automata. Theoretical Computer Science, 126(2):183-235, 1994.

2. R. Alur and P. Madhusudan. Decision problems for timed automata: A survey. In SFM-RT'04, volume 3185 of LNCS, pages 1-24, 2004.

3. G. Behrmann, A. David, K. G Larsen, J. Haakansson, P. Pettersson, W. Yi, and M. Hendriks. Uppaal 4.0. In QEST'06, pages 125-126, 2006.

4. B. Bérard, B. Bouyer, and A. Petit. Analysing the pgm protocol with UPPAAL. Int. Journal of Production Research, 42(14):2773-2791, 2004.

5. B. Berthomieu and M. Menasche. An enumerative approach for analyzing time petri nets. In IFIP Congress, pages 41-46, 1983.

6. A. Bouajjani, S. Tripakis, and S. Yovine. On-the-fly symbolic model checking for real-time systems. In RTSS'97, pages 25-, 1997.

7. P. Bouyer. Forward analysis of updatable timed automata. Formal Methods in System Design, 24(3):281-320, 2004.

8. M. Bozga, C. Daws, O. Maler, A. Olivero, S. Tripakis, and S. Yovine. Kronos: a mode-checking tool for real-time systems. In $C A V^{\prime} 98$, volume 1427 of $L N C S$, pages 546-550. Springer, 1998.

9. J.-M. Couvreur, A. Duret-Lutz, and D. Poitrenaud. On-the-fly emptiness checks for generalized büchi automata. In SPIN'05, volume 3639 of Lecture Notes in Computer Science, pages 169-184, 2005.

10. C. Daws and S. Tripakis. Model checking of real-time reachability properties using abstractions. In $T A C A S^{\prime} 98$, volume 1384 of $L N C S$, pages 313-329, 1998.

11. D. L. Dill. Timing assumptions and verification of finite-state concurrent systems. In Proc. Int. Workshop on Automatic Verification Methods for Finite State Systems, volume 407 of LNCS, pages 197-212. Springer, 1990.

12. F. Herbreteau, B. Srivathsan, and I. Walukiewicz. Efficient emptiness check for timed büchi automata. In $C A V^{\prime} 10,2010$. to appear.

13. S. Schwoon and J. Esparza. A note on on-the-fly verification algorithms. In TACAS'05, volume 3440 of $L N C S$, pages 174-190, 2005.

14. S. Tripakis. Verifying progress in timed systems. In Formal Methods for Real-Time and Probabilistic Systems, volume 1601 of LNCS, pages 299-314, 1999.

15. S. Tripakis. Checking timed büchi emptiness on simulation graphs. ACM Transactions on Computational Logic, 10(3), 2009.

16. S. Tripakis, S. Yovine, and A. Bouajjani. Checking timed büchi automata emptiness efficiently. Formal Methods in System Design, 26(3):267-292, 2005. 


\section{A Proofs}

\section{A.1 Proof of Theorem 3}

Proof. The basic Couvreur's algorithm has been proved in [13]. The Todo stack and the explore bit are the additions made to enable recursive splitting of SCCs. We first list below the invariants related to these new elements and prove them. In particular, we provide the proof that the calls to check_dfs made at line 16 and line 47 are correct.

Subsequently, we list all the invariants, and omit the proof details, which can be obtained based on the proof given in [13].

Let the latest call to $c h e c k \_d f s$ involved a node $s$ and a set blk of clocks.

1. Transitions containing clocks in blk are blocking transitions.

2. For a node $t$, t.explore $=$ true implies that $t$ was found in a maximal SCC $\Gamma$ that was explored with transitions upper-bounding clocks in $b l k_{\Gamma}$ removed and $\Gamma$ contained a set newblk $k_{\Gamma}$ of blocking clocks. So $t$ has to be explored with transitions in $b l k_{\Gamma} \cup$ newblk $k_{\Gamma}$ removed.

3. The Todo stack can be considered as a sequence of nodes interleaved with the $\$$ symbol: $\$^{0} s_{0}^{0} \ldots s_{i_{0}}^{0} \$^{1} \ldots \$^{n} s_{0}^{n} \ldots s_{i_{n}}^{n}$. We number the $\$$ symbols for clarity. Let $t^{i}$ be the node such that $\$^{i}$ was pushed during execution of line 43 of check_df $s\left(t^{i},-,-, b l k^{i}\right)$.

(a) $t^{i}$ is the root of a maximal SCC $\Gamma^{i}$ that does not contain any transition upper-bounding a clock in $b l k^{i}$.

(b) Every $\Gamma^{i}$ has a set newblk $k_{i}$ of blocking clocks. For $0 \leq i<n, b l k^{i+1}=$ $b l k^{i} \cup n e w b l k^{i}$ and $b l k=b l k^{n} \cup n e w b l k^{n}$.

(c) For $k \in\{0, \ldots, n\}$ and $j \in\left\{0, \ldots, i_{k}\right\}, s_{j}^{k}$.explore $=$ true implies that its explore bit was set to true during the execution of line 40 of check_df $s\left(t^{i},-,-, b l k^{i}\right)$.

(d) If there is a node $t_{\text {last }}$ last popped from the Todo stack whose check_df $s$ has not terminated, then there is a path from $t_{\text {last }}$ to $s$ not containing transitions upper-bounding clocks $b l k$.

(e) When $c h e c k_{-} d f s(s, \ldots)$ terminates, all the $\$$ symbols added during its visit are removed.

We first prove the invariants at line 12. Assume that the invariants are true before an iteration of the loop. The current node is $s$ and the successor obtained is $t$. There are three cases.

- If the condition in line 13 is satisfied, $t$ is pushed on to the Todo stack if it is not present already. Note that invariant 1 suggests that the transition from $s$ to $t$ contains a blocking clock. As the Todo stack is modified, we need to verify invariant 3 . It is sufficient to verify only when t.explore $=$ true.

The only change could occur in invariant $3(\mathrm{c})$. We now show that $t$ belongs to $\Gamma^{n}$, thus proving $3(\mathrm{c})$ after the addition of $t$ to Todo. Since invariant 3 is true before pushing $t$ to Todo stack, from invariant 3(c), $t_{\text {last }}$ belongs to $\Gamma_{n}$. From invariant $3(\mathrm{~d})$, there is a path from $t_{\text {last }}$ to $s$, not containing transitions 
upper-bounding clocks in blk. Hence this path does not contain transitions upper-bounding clocks in $b l k^{n}$ too. Therefore, the path from $t_{\text {last }}$ to $s$ would have been explored during the exploration of $\Gamma_{n}$. Since $t \notin$ Todo, $t$ would have been $c h e c k \_d f s(t, \ldots)$ would have been called during the exploration of $\Gamma_{n}$. Now if t.explore $=$ true, it clearly should belong to $\Gamma_{n}$.

- If the condition in line 15 is satisfied, we need to prove that the call at line 16 is correct. From an argument similar to above, we can infer that $t$ belongs to $\Gamma_{n}$. Invariant 2 and invariant $3(\mathrm{~b})$, the call at line 16 is correct.

- If the condition in line 17 is true, an SCC is detected and merge_scc is called. There is no change in the Todo stack and hence the invariants involving the Todo stack do not change.

We now prove the invariants at line 43. At 43, a maximal SCC with $s$ has root has been detected. This would be the $\Gamma^{n}$ of invariant 3 , with newblk $k^{n}=u b-r$. The $\$$ pushed now would be $\$_{n}$ of invariant 3 . The invariants are clearly true after the addition of a $\$$ and a node. At line 45 a node $s^{\prime}$ is removed from the Todo stack. From invariant 3, if $s^{\prime}$.explore is true, invariant 2 proves the correctness of the call made at 47 . We assume that after the call the invariants are true. From invariant $3(\mathrm{e})$, the same $\$_{n}$ is still the last $\$$ symbol. Therefore, the invariants are true after the loop at line 44 . The pop function performed at line 48 proves the invariant $3(\mathrm{e})$ after execution of line 48 . Clearly, the rest of the invariants remain unchanged at line 48.

We now list the other invariants below and omit their proofs.

1. For a node $s$, s.opened $=$ true iff $s$ has been visited and its SCC has not been completely closed yet.

2. The Roots stack is ordered by $d f$ snum. The Roots stack is a sequence of nodes $z_{0} \ldots z_{p} w_{0} \ldots w_{p^{\prime}}$. The sequence $z_{0} \ldots z_{p}$ represents the exploration with no blocking clocks. The sequence $w_{0} \ldots w_{p^{\prime}}$ is the result of detecting a maximal SCC $\Gamma$ which is blocked. The sequence $w_{0} \ldots w_{p^{\prime}}$ represents the re-exploration of a sub-SCC of $\Gamma$ with the set $b l k_{c u r r}$ of blocking clocks. Let $z_{\Gamma}$ be the root of $\Gamma$. Then $z_{\Gamma}$.df snum $>z_{p}$.df snum.

(a) For $0 \leq i<p$, the SCC of $z_{i}$ contains all nodes with $d f$ snum between $z_{i} . d f$ snum and $z_{i+1}$.df snum that have their opened set to true.

(b) The SCC of $z_{p}$ contains the nodes with $d f$ snum between $z_{\Gamma} . d f$ snum that have their opened set to true.

(c) For $0 \leq i<p^{\prime}$, the SCC of $w_{i}$, with transitions blocking clocks in $b l k$ removed, contains all nodes with $d f$ snum between $w_{i} . d f$ snum and $w_{i+1} . d f$ snum that have their opened set to true. The SCC of $w_{p^{\prime}}$ with blocking transitions due to clocks in blk removed contains the nodes with df snum greater than $w_{p^{\prime}}$.df snum that have their opened set to true.

(d) Every $z_{i}$ contains a set $u b$ of clocks that are upper-bounded and a set $r$ of clocks that are reset in its SCC found so far. Every $w_{i}$ again contains these two sets.

3. The Active stack contains the set of nodes whose opened bit is true. It is ordered by the order of appearance of the states during exploration. 
4. Line 32 of the algorithm is executed iff an accepting unblocked SCC is found.

Complexity: A call check_df $s(s, \ldots)$ is made only when s.explore is true. For a node $s$, the explore bit is initially assumed to be true. Thus check_df $s(s, \ldots)$ is first called when $s$ is first visited. It sets s.explore to false in line 9 . Future assignments of explore to true occur in line 40 when a maximal SCC being closed has more blocking clocks. Let this set of blocking clocks be blk and this maximal SCC be $\Gamma$. If $\Gamma$ is completely closed without setting s.explore to true, then s.explore can never be set to true again. Hence, if s.explore becomes true again, it can be inferred that $\Gamma$ is still being explored and s.explore is set while closing a maximal SCC $\Gamma^{\prime} \subset \Gamma$ with a set $b l k^{\prime} \neq \emptyset$ of more blocking clocks. Therefore the next call to $\operatorname{check}_{-} d f s(s, \ldots)$ occurs $\Gamma^{\prime}$ is explored with the set $b l k \cup b l k^{\prime}$ of blocking clocks. Each time $\operatorname{check}_{-} d f s(s, \ldots)$ is called with a strictly increasing set of blocking clocks. Thus the number of calls to check_df $s(s, \ldots)$ for every node $s$ is bounded by $|X|$. The size of $G Z G(\mathcal{A})$ is $\mathcal{O}(|Z G(\mathcal{A})| \cdot|X|)$. Hence the complexity of the algorithm is $\mathcal{O}\left(|Z G(\mathcal{A})| \cdot|X|^{2}\right)$.

\section{A.2 Proof of Lemma 1}

Proof. Consider a path in $Z G(\mathcal{A})$ and one instance of the path:

$\left(q_{0}, Z_{0}\right) \cdots \stackrel{\{t\}}{\longrightarrow}\left(q_{i_{1}}, Z_{i_{1}}\right) \cdots\left(q_{i_{1}^{\prime}}, Z_{i_{1}^{\prime}}\right) \stackrel{t \geq 1}{\longrightarrow} \cdots \stackrel{\{t\}}{\longrightarrow}\left(q_{i_{k}}, Z_{i_{k}}\right) \cdots\left(q_{i_{k}^{\prime}}, Z_{i_{k}^{\prime}}\right) \stackrel{t \geq 1}{\longrightarrow} \cdots$ $\left(q_{0}, \mathbf{0}\right) \cdots \stackrel{\{t\}}{\longrightarrow}\left(q_{i_{1}}, \nu_{i_{1}}\right) \cdots\left(q_{i_{1}^{\prime}}, \nu_{i_{1}^{\prime}}\right) \stackrel{t \geq 1}{\longrightarrow} \cdots \stackrel{\{t\}}{\longrightarrow}\left(q_{i_{k}}, \nu_{i_{k}}\right) \cdots\left(q_{i_{k}^{\prime}}, \nu_{i_{k}^{\prime}}\right) \stackrel{t \geq 1}{\longrightarrow} \cdots$

For every $j \geq 1$, we have $\nu_{i_{j}}(t)=0$ and $\nu_{i_{j}^{\prime}}(t) \geq 1$. It comes that the time that elapses on the run between steps $i_{j}$ and $i_{j}^{\prime}$ is at least 1 . Hence, the accumulated time over all steps $j$ diverges since there are infinitely many such $j$. As a consequence, the time that elapses on the run diverges.

\section{A.3 Proof of Lemma 3}

Recall Lemma 3:

"If a reachable SCC in $Z G(\mathcal{A})$ is unblocked, free from zero-checks and accepting, then $\mathcal{A}$ has a non-Zeno run."

Proof. The proof is similar to Lemma 4.13 in [1]. Consider an infinite path $\pi$ in the SCC of $Z G(\mathcal{A})$ that is unblocked, free from zero-checks and accepting. Assume that all its instances are Zeno. Consider one particular instance $\rho$. Let $X_{0}$ be the clocks that are reset infinitely often on $\rho$. Consider a suffix $\rho^{\prime}$ of $\rho$ such that:

- the total amount of time that elapse on $\rho^{\prime}$ is less than 1 ;

- we can identify an infinite number of configurations $\left(q_{i}, \nu_{i}\right)$ on $\rho^{\prime}$ such that:

- all the clocks from $X_{0}$ are reset between $\left(q_{i}, \nu_{i}\right)$ and $\left(q_{i+1}, \nu_{i+1}\right)$ for all $i$; 
- the time that elapses between $\left(q_{i}, \nu_{i}\right)$ and $\left(q_{i+1}, \nu_{i+1}\right)$ is less than 0.5.

Then, the value of the clocks outside of $X_{0}$ satisfy all the guards of the transitions on $\rho^{\prime}$. The value of all the clocks from $X_{0}$ is always less than 0.5.

Now, we build a non-Zero run from $\rho$ in the following way. In every configuration $\left(q_{i}, \nu_{i}\right)$ we decide to let time elapse for 0.5 time unit. This is still a run of $\mathcal{A}$ since:

- no clock is zero-check on $\rho$ by definition of $\pi$;

- the value of all the clocks from $X_{0}$ is still less than 1 , hence they satisfy the same guards;

- no clock is blocking.

We deduce that $\mathcal{A}$ has a non-Zeno run.

\section{A.4 Proof of Lemma 4}

We recall Lemma 4:

"Let $\Gamma$ be a reachable maximal SCC in $Z G(\mathcal{A})$, with root $\left(q^{\Gamma}, Z^{\Gamma}\right)$. $\mathcal{A}$ has an accepting non-Zeno run ending in $\Gamma$ iff $G Z G(\mathcal{A})_{\mid \Gamma}$ has an unblocked accepting SCC reachable from $\left(q^{\Gamma}, Z^{\Gamma}, X\right)$."

Proof. Let $G Z G(\mathcal{A})_{\mid \Gamma}$ be the part of $G Z G(\mathcal{A})$ restricted to nodes and transitions that occur in $\Gamma$. More precisely, the nodes of $G Z G(\mathcal{A})_{\mid \Gamma}$ are all the nodes $(q, Z, Y)$ of $G Z G(\mathcal{A})$ such that $(q, Z)$ is a node in $\Gamma$. The transitions of $G Z G(\mathcal{A})_{\mid \Gamma}$ are all the transitions of $G Z G(\mathcal{A})$ that connect nodes in $G Z G(\mathcal{A})_{\mid \Gamma}$.

We prove that there is an SCC in $G Z G(\mathcal{A})_{\mid \Gamma}$ that is unblocked and accepting iff it is reachable from $\left(q^{\Gamma}, Z^{G}, X\right)$.

By definition of $G Z G(\mathcal{A})_{\mid \Gamma}$, every node reachable from $\left(q^{\Gamma}, Z^{\Gamma}, X\right)$ in $G Z G(\mathcal{A})_{\mid \Gamma}$ is also reachable from $\left(q^{\Gamma}, Z^{\Gamma}, X\right)$ in $G Z G(\mathcal{A})$. From $(1)$, we also have that $\left(q^{\Gamma}, Z^{G}, X\right)$ is reachable in $G Z G(\mathcal{A})$.

$$
(q, Z) \stackrel{g ; R}{\longrightarrow}\left(q^{\prime}, Z^{\prime}\right) \quad \text { iff } \quad(q, Z, X) \stackrel{g ; R}{\longrightarrow}\left(q^{\prime}, Z^{\prime}, X\right)
$$

Hence, if an SCC is reachable in $G Z G(\mathcal{A})$ from $\left(q^{\Gamma}, Z^{\Gamma}, X\right)$, it is also reachable from the initial node of $G Z G(\mathcal{A})$. By Theorem $2 \mathcal{A}$ has a non-zeno accepting run (ending in $\Gamma$ ).

It remains to prove that if $\mathcal{A}$ has an accepting non-Zeno run ending in $\Gamma$, then $G Z G(\mathcal{A})$ has an unblocked accepting SCC reachable in $G Z G(\mathcal{A})_{\mid \Gamma}$. Consider the suffix $\rho$ of the non-Zeno accepting run of $\mathcal{A}$ such that all its transitions are in $\Gamma$. It is thus an instance of a path in $\Gamma$. By Theorem 2 there exists a unblocked path $\pi$ in $G Z G(\mathcal{A})$ that visits both a clear node and an accepting node infinitely often, and that abstract $\rho$. By definition, $G Z G(\mathcal{A})_{\mid \Gamma}$ contains $\pi$.

The nodes that occur infinitely often on $\pi$ define an SCC $\Gamma^{\prime}$ in $G Z G(\mathcal{A})_{\mid \Gamma}$. There exists a clear node $(q, Z, \emptyset)$ in $\Gamma^{\prime}$. By definition, $(q, Z, \emptyset)$ is reachable from $(q, Z, X)$ by a $\tau$-transition. By $(1),\{(q, Z, X) \mid(q, Z) \in \Gamma\}$ is an SCC. Hence $\left(q^{\Gamma}, Z^{\Gamma}, X\right)$ can reach $(q, Z, X)$ and $(q, Z, \emptyset)$. We have an unblocked (recall $\pi$ is unblocked) SCC that contains both an accepting node and a clear node that is reachable from $\left(q^{\Gamma}, Z^{\Gamma}, X\right)$. 


\section{B.2 Full On-The-Fly Emptiness Check Algorithm for Timed Büchi Automata}

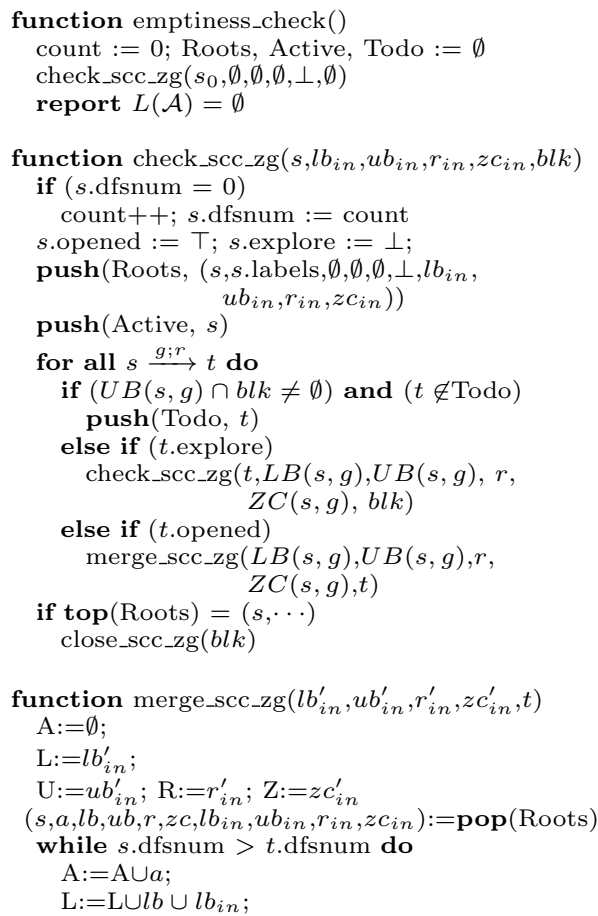

$\mathrm{U}:=\mathrm{U} \cup u b \cup u b_{i n} ; \mathrm{R}:=\mathrm{R} \cup r \cup r_{i n} ;$

$\mathrm{Z}:=\mathrm{Z} \vee z c \vee z c_{i n}$

$\left(s, a, l b, u b, r, z c, l b_{i n}, u b_{i n}, r_{i n}, z c_{i n}\right):=\operatorname{pop}($ Roots)

$\mathrm{A}:=\mathrm{A} \cup a ; \mathrm{L}:=\mathrm{L} \cup l b ; \mathrm{U}:=\mathrm{U} \cup u b ; \mathrm{R}:=\mathrm{R} \cup r ; \mathrm{Z}:=\mathrm{Z} \cup z c$

push(Roots, $\left.\left(s, \mathrm{~A}, \mathrm{~L}, \mathrm{U}, \mathrm{R}, \mathrm{Z}, l b_{i n}, u b_{i n}, r_{i n}, z c_{i n}\right)\right)$

if $(A c c \subseteq A)$ and $((L \cap R \neq \emptyset)$ or $((U \subseteq R)$ and not $Z))$ report $L(\mathcal{A}) \neq \emptyset$

function close_scc $\_\mathrm{zg}(b l k)$

$\left(s, a, l b, u b, r, z c, l b_{i n}, u b_{i n}, r_{i n}, z c_{i n}\right):=\operatorname{pop}($ Roots $)$

$\mathrm{SCC}:=\emptyset$

repeat

$u:=\operatorname{pop}($ Active $)$

$u$.opened $:=\perp$

if $(\operatorname{Acc} \subseteq a)$ and $(u b \not r)$ $u$.explore $:=\top$

else if $(\operatorname{Acc} \subseteq a)$ and $(z c)$

$u$.explore $:=\top$

$\operatorname{push}(\mathrm{SCC}, u)$

until $u=s$

if $(\operatorname{Acc} \subseteq a)$ and $(u b \not \subset r)$

push(Todo, $\$$ ); push(Todo, $s$ )

while top $($ Todo $) \neq \$$ do

$s^{\prime}:=\operatorname{pop}($ Todo)

if $\left(s^{\prime}\right.$.explore $)$

check_scc_zg $\left(s^{\prime}, \emptyset, \emptyset, \emptyset, \perp, b l k \cup(u b-r)\right)$

$\operatorname{pop}($ Todo)

else if $(\operatorname{Acc} \subseteq a)$ and $(z c)$

check_scc_gzg $((s, X), \emptyset, \emptyset, b l k)$

repeat

$u:=\operatorname{pop}(\mathrm{SCC}) ; u$.explore $:=\perp$

until $\mathrm{SCC}=\emptyset$

Fig. 6. On-The-Fly Emptiness Check Algorithm on the Zone Graph. 


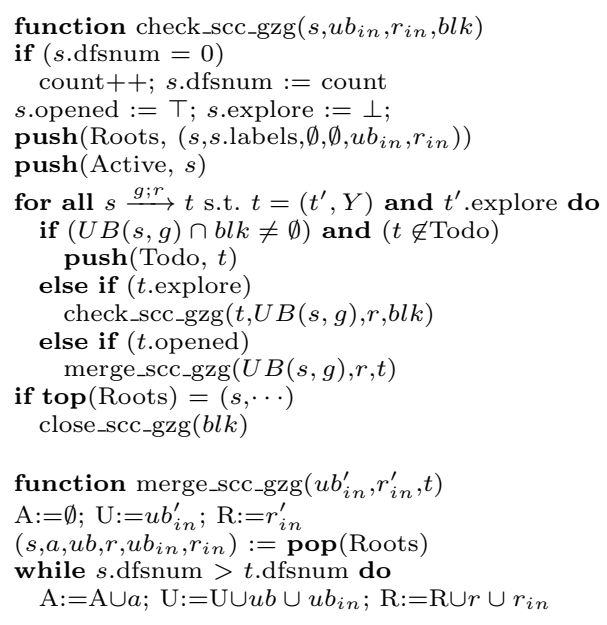

Fig. 7. On-The-Fly Emptiness Check Functions on the Guessing Zone Graph. 